\title{
SUMO-specific proteases/isopeptidases: SENPS and beyond
}

\author{
Arnab Nayak and Stefan Müller
}

\begin{abstract}
We summarize the evolutionary relationship, structure and subcellular distribution of SUMO proteases (or SUMO isopeptidases). We also discuss their functions and allude to their involvement in human disease.
\end{abstract}

Keywords: SUMO, SUMO isopeptidase, SUMO-specific protease, SENP, Desi-1, Desi-2, USPL1

\section{Introduction}

The ubiquitin-like SUMO (small ubiquitin-related modifier) system is a post-translational protein modification pathway in eukaryotes [1,2]. SUMOylation is a highly dynamic process, where deconjugation (deSUMOylation) is catalyzed by a family of cysteine proteases, termed SUMO-specific proteases or SUMO isopeptidases. A subset of these enzymes also functions in processing of the SUMO precursor proteins, which is a prerequisite for their conjugation. In this review, we summarize the current view of SUMO deconjugating enzymes. We discuss their evolutionary relationships, subcellular distributions and functions as well as their involvement in human disease.

SUMO belongs to the family of ubiquitin-like proteins. Like ubiquitin, it functions as a protein modifier that is covalently attached to $\varepsilon$-amino groups of lysine residues of target proteins $[1,2]$. Lower eukaryotes attach a single SUMO form (known as smt3) to target proteins, whereas the human SUMO system makes use of three related SUMO forms (SUMO1, SUMO2 and SUMO3). All SUMO/smt3 forms are expressed as precursor proteins that require carboxy-terminal proteolytic processing prior to their conjugation. Processing exposes a carboxy-terminal di-glycine motif, which is essential for conjugation. Subsequent conjugation proceeds by a pathway that typically involves an E1-E2-E3 enzymatic

\footnotetext{
* Correspondence: ste.mueller@em.uni-frankfurt.de Institute of Biochemistry II, Goethe University, Faculty of Medicine, Theodor-Stern-Kai 7, 60590 Frankfurt, Germany
}

cascade. Modification by SUMO generally controls protein-protein interactions by recruiting binding partners that harbor specific SUMO-interaction motifs (SIMs).

Regulated deconjugation of SUMO from its substrates is a central element of the SUMO system as it assures the plasticity of protein interaction networks. The deconjugation process is catalyzed by a family of cysteine proteases, termed SUMO isopeptidases or SUMO-specific proteases. Members of this enzyme class function as deconjugating enzymes for isopeptide-linked SUMO-protein conjugates and also depolymerize isopeptide-linked poly-SUMO2/3 chains. Moreover, some family members act as processing factors for the carboxy-terminal maturation of the SUMO precursor. The known SUMO-specific isopeptidases and proteases are cysteine proteases that are classified into three distinct families: the Ulp/SENP (ubiquitin-like protease/sentrin-specific protease) family, the Desi (deSUMOylating isopeptidase) family and USPL1 (ubiquitin-specific peptidase-like protein 1).

\section{Gene organization and evolutionary history}

All identified SUMO isopeptidases/proteases are cysteine proteases. They share a similar catalytic mechanism but belong to different superfamilies that are distinguished by the fold of their respective catalytic domains.

Ulp/SENP proteins belong to the C48 family subgroup of the CE superfamily of thiol proteases whose founder member is Ulp1, first discovered in the yeast Saccharomyces cerevisiae [3]. Subsequently, Ulp2 was identified as a second SUMO-deconjugating enzyme in yeast [4]. Ulp1/2-related isopeptidase genes are also found in the genome of Drosophila melanogaster [5,6]. In higher eukaryotes, the family is more diverse. Seven human proteins were initially classified as Ulp family members and annotated as SENPs [7]. (The term sentrin was coined by Ed Yeh as an alternative name for SUMOs.) Notably, however, later experimental work revealed that SENP8 acts on the ubiquitin-family member Nedd8, but not on SUMO paralogs $[8,9]$. The human genome therefore 
encodes six dedicated SUMO-specific members of the Ulp/SENP family: SENP1, SENP2, SENP3, SENP5, SENP6 and SENP7. Sequence analyses and phylogenetic comparison of the human SENPs with Ulp1 and Ulp2 from lower eukaryotes illustrate that SENP1, SENP2, SENP3 and SENP5 are evolutionarily related to the Ulp1 branch, whereas SENP6 and SENP7 are related to the Ulp2 branch (Figure 1). The tree further reveals the pairwise similarity of SENP1 to SENP2, SENP3 to SENP5 and SENP6 to SENP7.

The deSUMOylating isopeptidases Desi-1 and Desi-2 belong to the evolutionarily distinct C97 family of cysteine proteases [10]. Orthologs of Desi-1 and Desi-2 are found in plants and metazoa, but are missing in lower eukaryotes such as yeast.

USPL1 is, to date, the only known mammalian SUMO-specific protease of the C98 family [11]. USPL1related deconjugases are found in metazoan vertebrates and invertebrates. USPL1 is not related to the Ulp/SENP and Desi families, but the catalytic domain of USPL1 shows homology to the C19 family of ubiquitin-specific proteases. For example, within this region, USPL1 shares around $20 \%$ sequence identity with the ubiquitindeconjugating enzyme USP1.

\section{Characteristic structural features}

The common characteristic of the Ulp/SENP family is their conserved catalytic domain, which spans around 200 amino acids in the carboxy-terminal part of the protein (Figure 2). This domain has sequence and structural similarity to the catalytic region of adenoviral proteases, which cleave viral and cellular proteins. The human Ulp/SENP family members share 20 to $60 \%$ sequence identity within their catalytic domains. The SENP1-

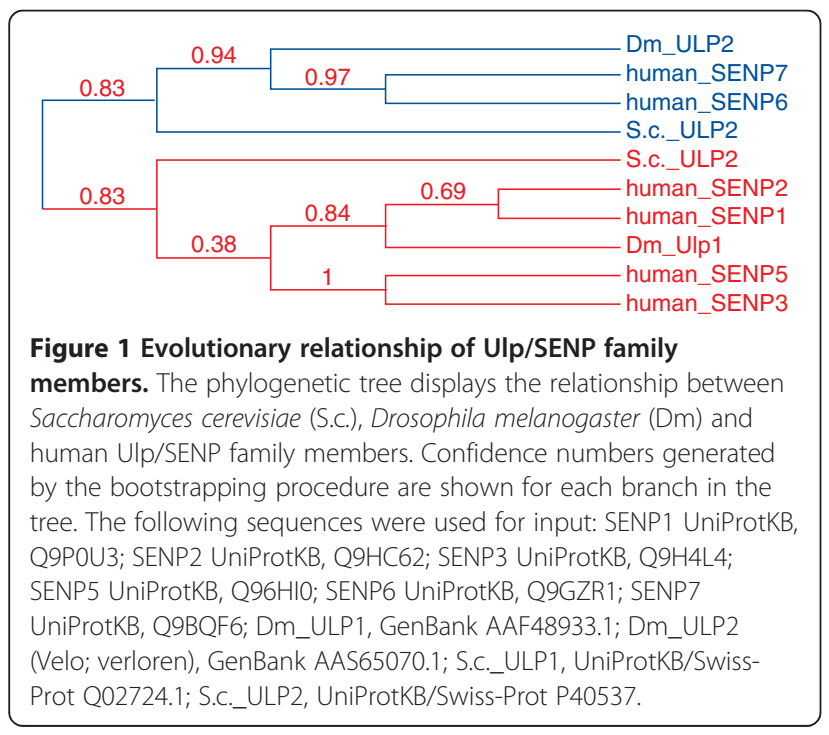

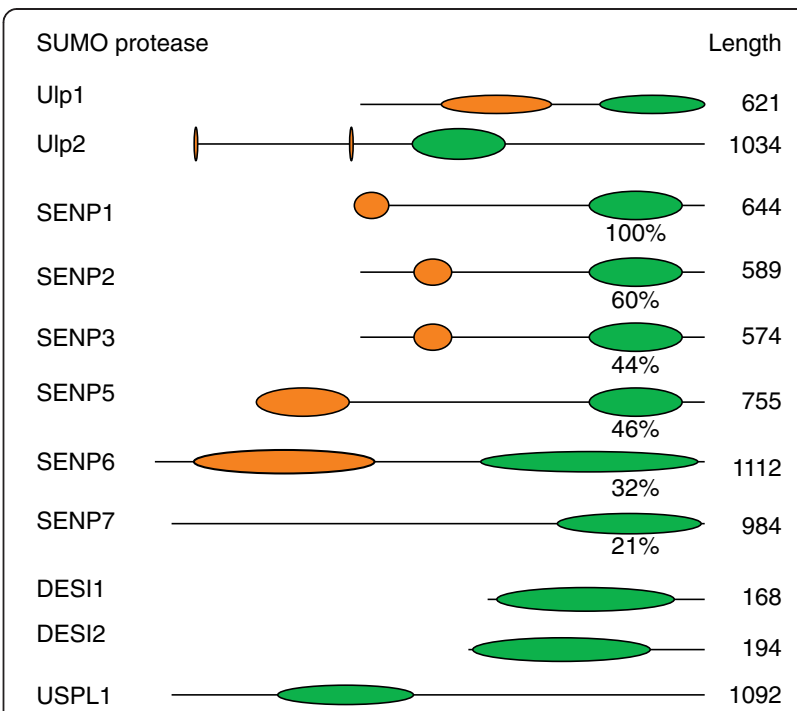

Figure 2 Structural organization of SUMO-specific-proteases/ isopeptidases. The domain organizations of Ulp/SENPS and Desi family members are shown. Green ovals represent the catalytic domain. The sequence determinants that are responsible for subcellular targeting are represented by orange ovals. The length of the proteins as total number of amino acids is presented on the right side. For the catalytic domains of SENP family members, sequence identity shared with SENP1 is also shown.

SENP2, SENP3-SENP5 and SENP6-SENP7 pairs show the highest degree of similarity to each other. SENP6 and SENP7 are most divergent from the rest of the family and harbor conserved sequence insertions within their catalytic domains. Structural data describing the catalytic domain of yeast Ulp1 and human SENP1 and SENP2 in complex with SUMO precursors or isopeptide-linked SUMO-RanGAP1 conjugates revealed important insights into the catalytic features of this enzyme class [12-16]. The active site cysteine residue is embedded in a typical catalytic triad (Cys-His-Asp). An additional invariant Gln residue in close proximity stabilizes the transition state during catalysis. The substrate accesses the catalytic site through a shallow tunnel, in which conserved Trp residues are essential for positioning the di-glycine motif and the scissile bond over the active site. A remarkable finding was that the scissile bond of the pre-SUMO variants or a SUMO-conjugate is oriented in a cis configuration for cleavage [14,15].

In addition to their conserved catalytic domain, all SENPs have amino-terminal regions of variable length that have crucial regulatory functions. These regions frequently contain interaction domains for cellular adaptor proteins that determine the subcellular distribution of Ulp/SENP family members [17-19]. Post-translational modifications, such as phosphorylation or ubiquitylation, within these regions provide additional regulatory layers for the recruitment of binding partners or the control of 
Ulp/SENP stability [20-23]. Interestingly, most SENPs contain one or more SIM modules in their aminoterminal region, which probably contribute to the selection of substrates or facilitate the targeting of specific SENPs to poly-SUMO chains.

Desi-1 and Desi-2 are small proteins characterized by PPPDE (permutated papain fold peptidases of the double-stranded RNA viruses and eukaryotes) domains of around 140 amino acids. Desi-1 and Desi-2 share about $20 \%$ sequence identity within this region. Structural data from Desi-1 revealed that the protein forms a homodimer, in which the groove between the two subunits forms the active site. This region contains two conserved cysteine and histidine residues that form a catalytic dyad [10,24]. Interestingly, the active-site groove of Desi-1 is occupied by its own carboxyterminal segment [24], which is very different from the open cleft in SENPs.

The catalytic domain of USPL1 contains a catalytic triad composed of Cys-His-Asp-residues [11,25].

\section{Localization}

The different SUMO isopeptidases have characteristic subcellular distributions, which seems to be a way of restricting their activity to a specific set of substrates. The Ulp/SENP family members are predominantly concentrated in distinct subnuclear regions (Figure 3). SENP1 and SENP2, as well as the yeast Ulp1 enzyme, are concentrated at the nuclear envelope through their interaction with components of the nuclear pore complex [18,26-28]. Within the nucleus SENP1 and SENP2 are excluded from the nucleolus, but enriched in nuclear foci that partially overlap with PML nuclear bodies. In mitosis, SENP1 and SENP2 redistribute from the nuclear envelope to the kinetochore [29]. It is worth noting that despite its predominately nuclear localization, SENP2 was reported to shuttle between the nucleus and the cytoplasm [30]. Moreover, distinct splice variants of SENP2 may exhibit specific subcellular distributions [31].

SENP3 and SENP5 are compartmentalized in the nucleolus, where they act on proteins that are involved in the early steps of ribosome maturation [17,32-34]. A subfraction of SENP3 and SENP5 also resides in the nucleoplasm and the cytoplasm. Interestingly, at $G_{2} / M$ transition, prior to nuclear envelope breakdown, SENP5 translocates to the mitochondrial surface [35]. SENP6 and SENP7 mainly exhibit a nucleoplasmic distribution. In contrast to SENPs, Desi family members are primarily concentrated in the cytoplasm [35]. USPL1 is a predominantly nuclear protein and co-localizes with coilin in Cajal bodies $[11,25]$.

\section{Function}

SUMO maturation versus SUMO deconjugation

Ulp/SENPs can act as deconjugating or maturation/processing enzymes. The yeast Ulp family was covered in a recent review [31] and will not be further discussed here. Distinct human SENPs exert different activities in either processing or deconjugation (Figure 4). Moreover, preferences for specific SUMO paralogs have been reported for specific SENPs as outlined below. The maturation process removes the amino acids carboxy-terminal to a di-glycine motif, whose exposure is essential for conjugation. The substrate specificity of SENPs for processing is
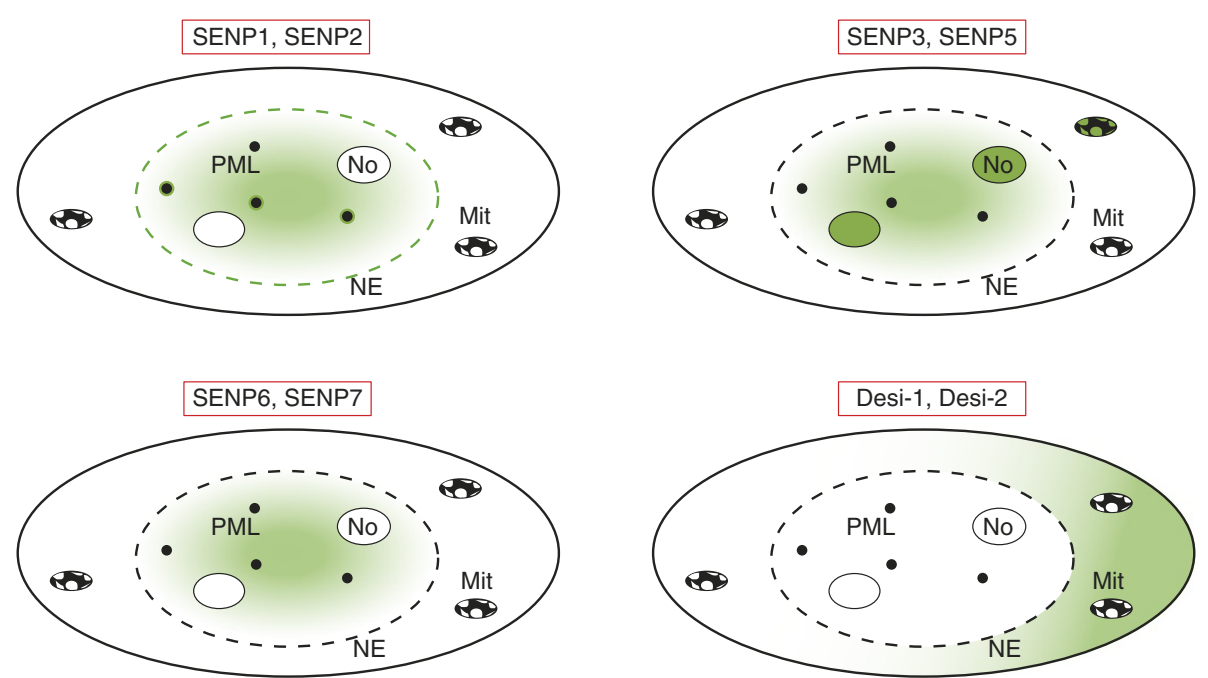

Figure 3 Schematic representation of the subcellular distribution of mammalian SUMO-specific isopeptidases of the SENP and Desi families. The predominant subcellular distribution of the respective SENPs is indicated by the green color. Mit, mitochondria; NE, nuclear envelope; No, nucleolus; PML, promyelocytic leukemia nuclear bodies. 


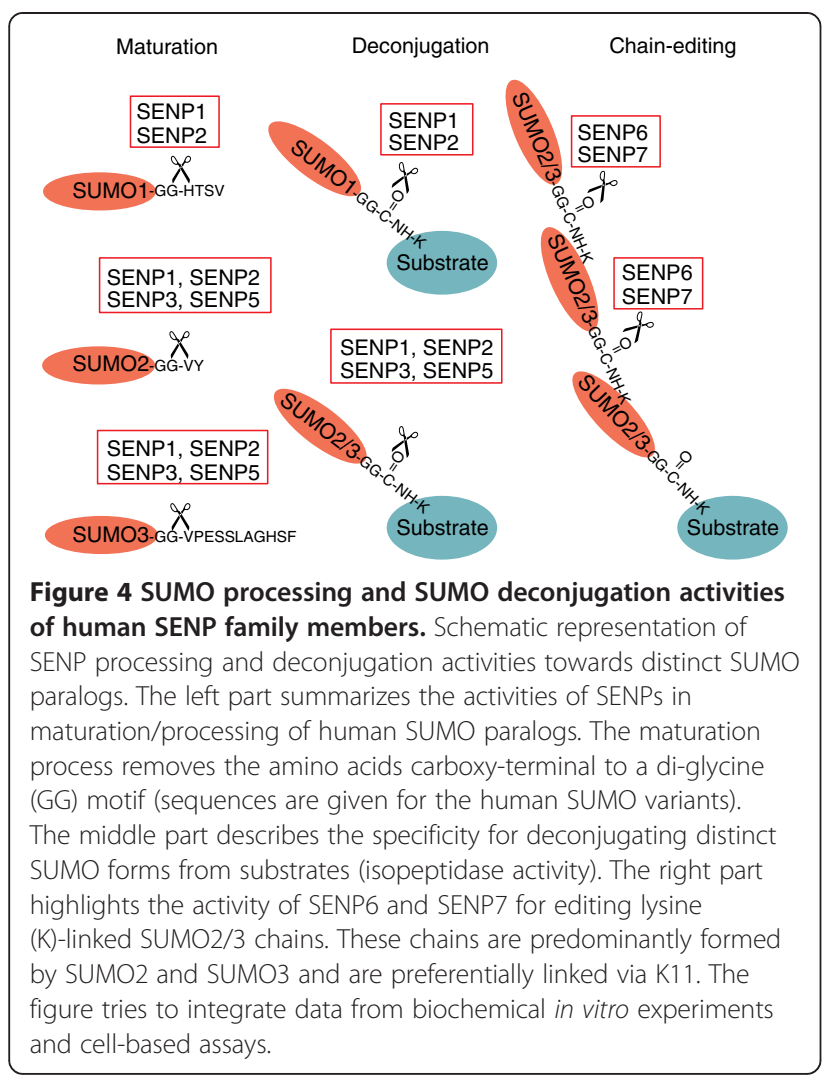

determined by the amino acids that are found carboxyterminal to the di-glycine motif.

Biochemical experiments have established a role for SENP1 in both SUMO deconjugation and SUMO processing. The data show that SUMO-1 is processed most efficiently, followed by SUMO-2 and SUMO-3. By contrast, at least in vitro, SENP1 displayed little specificity in its ability to deconjugate the different SUMO paralogs from SUMOmodified substrates $[13,15,16]$. Recent genetic data from mice, however, suggest that in vivo SENP1 has only a limited role in SUMO-2 and SUMO-3 deconjugation, but is essential for deSUMOylating SUMO1-modified proteins [36]. Biochemical experiments demonstrate that SENP2 catalyzes SUMO deconjugation more efficiently than SUMO processing and is most efficient for SUMO2 over SUMO1 and SUMO3 $[14,37,38]$. The SENP3-SENP5 couple shows a very strong preference for processing and deconjugation of SUMO2 and SUMO3 and does not exhibit significant processing and deconjugase activity for SUMO1 [32,39]. SENP6 and SENP7 exert only very weak processing activity and are also rather inefficient in deconjugating monomeric SUMO from substrates. Importantly, however, SENP6 and SENP7, as well as the related Ulp2 from yeast, are excellent enzymes for deconjugating SUMO moieties from SUMO2-SUMO3 di-SUMOylated substrates and from polymeric chains of SUMO2 and SUMO3 [39-42]. Their main function seems to be the editing of lysine-linked SUMO-SUMO chains.
In contrast to Ulp/SENP family members, the Desi-1 and Desi-2 enzymes exert isopeptidase activity on a selected substrate, but have only an extremely low processing activity for the pre-SUMO precursor protein [10,24]. USPL1 is active in processing and deconjugation.

\section{Substrate specificity of SUMO proteases}

With only nine SUMO isopeptidases identified to date, the SUMO-deconjugating machinery appears to be less complex than the de-ubiquitylating system. Even if there are unidentified SUMO isopeptidases or additional alternative splice variants of SENPs, their total number is likely to remain far below the currently known 100 ubiquitin deconjugases. The situation is similar when comparing the number of E3 ubiquitin ligases and E3 SUMO ligases. Why the modification and demodification of hundreds of different SUMO conjugates are controlled by only a limiting set of enzymes is a central question in the field. One scenario is that the SUMO system, including the deconjugation machinery, coordinately regulates groups of proteins that are functionally and physically linked [43]. In this scenario, a given SUMO isopeptidase is likely to act on a larger set of proteins, which in many cases are associated in larger protein complexes. For example, the human SENP3 enzyme deconjugates a number of SUMO2/3 conjugates at nucleolar pre-60S ribosomes [33,34,44-47]. The concept of group modification or demodification complicates the interpretation of experimental approaches that had concentrated on individual SUMO substrates for a given isopeptidase. In the following, we will try to integrate the available data to provide a more general picture and to define cellular pathways that are controlled by a distinct SUMO isopeptidase.

\section{Cellular pathways regulated by SUMO-specific isopeptidases SENP1}

The inactivation of transcription factors by SUMO conjugation and their activation by SUMO deconjugation is a recurrent theme. SENP1-mediated deSUMOylation seems to be crucial for the activation of transcriptional programs in innate immune responses and in the development of B and $\mathrm{T}$ cells. In these processes, IRF8, STAT5 and Bcl11b have been defined as relevant SENP1 targets [48-50], but SENP1 probably acts on a broader spectrum of transcription factors in these pathways and other processes. Along this line, it has been shown that deSUMOylation of HIF1 $\alpha$ by SENP1 under conditions of hypoxia is required for stabilization of HIF1 $\alpha$ and the expression of HIF1 $\alpha$ target genes. Recruitment of SENP1 to specific substrates could be coordinated by posttranslational modifications as exemplified by the phospho-dependent binding of SENP1 to Bcl11b [50]. 
In mitotic cells, SENP1 appears to target selected substrates at the kinetochore. This is critical for mitotic progression because the knockdown of SENP1 delays sister chromatid separation at metaphase [29].

\section{SENP2}

Like SENP1, SENP2 is involved in the regulation of gene expression programs in developmental processes. Yeh and co-workers [51] demonstrated that deletion of the SENP2 gene in mice causes defects in cardiac development resulting from the reduced expression of Gata4 and Gata6. This reduced expression has been linked to the lack of SENP2mediated deSUMOylation of a subunit of the polycomb repressive complex 1 (PRC1). PRC1 represses transcription of Gata4, Gata6 and numerous other developmental regulator genes, indicating a central role for SENP2 in early embryonic development.

\section{SENP3}

SENP3 has a well-established function in the control of ribosome biogenesis and particularly affects the maturation of the $28 \mathrm{~S}$ rRNA [33,34]. This is likely to involve the SENP3-catalyzed removal of SUMO2 or SUMO3 from various $60 \mathrm{~S}$ maturation factors acting on nucleolar pre-60S ribosomal particles. In addition to its role in ribosome biogenesis, a nucleoplasmic subfraction of SENP3 controls transcriptional processes through demodification of transcriptional co-regulators and components of chromatin-modifying complexes [52]. Recent data show that SENP3-mediated deSUMOylation controls the expression of osteogenic differentiation factors and of other developmental regulators through deSUMOylation of MLL1/2 histone-methyltransferase complexes [53].

SENP3 abundance and subcellular distribution are tightly regulated by environmental stimuli. Redoxsensitive cysteine residues in the amino-terminal region trigger the stabilization of SENP3 in response to redox stress, whereas the PERK1 kinase pathway induces its lysosomal degradation in response to oxygen or glucose deprivation $[20,54]$. In mitosis, SENP3 is heavily phosphorylated, which may control its substrate specificity and/or subcellular distribution [21].

\section{SENP5}

The nucleolar function of SENP5 is also related to the ribosome biogenesis pathway, where it is involved in RNA polymerase I-mediated transcription of the $47 \mathrm{~S}$ rRNA [34]. As mentioned above, upon $\mathrm{G}_{2} / \mathrm{M}$ transition, SENP5 translocates to mitochondria, where the deSUMOylation of mitochondrial proteins seems to drive mitochondrial fragmentation during mitosis $[35,55]$.

\section{SENP6}

SENP6 is the principal chain-editing enzyme in human cells and accordingly regulates multiple signaling pathways that are controlled by poly-SUMOylation. A well-characterized poly-SUMO2/3-regulated process is SUMO-mediated ubiquitylation by SUMO-targeted ubiquitin-ligases (StUbLs). StUBLs, whose prototypic member is RNF4, are polySUMO2/3-binding ubiquitin ligases that are recruited to poly-SUMOylated substrates to trigger their ubiquitylation. Among the established targets of RNF4 are the promyelocytic leukemia (PML) protein and the inner kinetochore protein CENP-I. SENP6 counterbalances this poly-SUMO-RNF4-dependent degradation pathway by preventing the polySUMOylation of PML or CENP-I, as evidenced by the accumulation of both proteins in the absence of SENP6 [56-58]. Other defined substrates for SENP6 are the NF- $\mathrm{KB}$ regulator NEMO and the replication factor RPA70 $[59,60]$. How SENP6 is selectively targeted to its substrates under specific conditions is currently not understood.

\section{SENP7}

Similar to SENP6, SENP7 preferentially acts on SUMOSUMO chains. Small interfering RNA (siRNA)-mediated SENP7 depletion experiments point to the crucial involvement of SENP7 in chromatin remodeling and chromatin dynamics. It has been proposed that chromatin relaxation in response to DNA damage is promoted by SENP7-mediated removal of SUMO2/3 chains from the KRAB-associated protein 1 (KAP1) [19]. This allows the recruitment of the chromatin remodeler CHD3, which triggers chromatin relaxation. Importantly, the heterochromatin protein HP1 seems to function as the chromatin-targeting adaptor for SENP7 and may itself be a substrate for SENP7. Interestingly, recent work identified a shorter splice variant of SENP7, which lacks the HP1 binding domain and accordingly is unable to deSUMOylate HP1 [61]. These data exemplify the possible role of alternative splicing of SENPs for substrate selection.

\section{Desi-1/2 and USPL1}

Only limited functional data on Desi-1/2 and USPL1 are currently available. Desi- 1 and -2 seem to have a more restricted substrate specificity, with the transcriptional repressor BZEL being the only substrate identified to date [10]. Consistent with its localization to Cajal bodies, USPL1 has been shown to be important for small nuclear ribonucleic particle (snRNP) assembly and pre-mRNA splicing, but the relevant substrates have not yet been identified [11,25].

\section{Frontiers}

Among the most burning questions in the field is the target specificity of distinct SUMO isopeptidases. Quantitative mass-spectrometry approaches that monitor 
SUMOylation in cells or tissues in which specific family members are depleted might be one promising approach to answer this question. Unraveling the substrate specificity of distinct SENP splice variants would also be an important aspect along this line. Considering that USPL1 exerts essential non-catalytic functions [11], it is also crucial to define potential functions of other SUMO isopeptidases that are not linked to their catalytic activity. Knock-in mice expressing catalytic-dead variants of the respective enzymes would be the best model system to tackle this search.

Future work will also need to uncover how the misregulation of SENPs is linked to human disease. Most studies have focused on an involvement of SENPs in the development and/or progression of cancer [62]. Overexpression of SENP1 has been correlated with prostate cancer aggressiveness and metastatic potential [63-65]. This is at least partially mediated through induction of HIF1 $\alpha$-dependent signaling pathways. SENP1 additionally activates other oncogenic signaling pathways, such as c-Jun and androgen-receptor-mediated transcription. Like SENP1, SENP3 accumulates in several human cancers, with colon carcinomas having the highest ratio of SENP3 expression $[54,66]$. These and other data indicate a potential significance for SENPs as diagnostic markers and also make this enzyme class an attractive drug target in distinct human tumors $[67,68]$.

\section{Abbreviations \\ Desi: deSUMOylating isopeptidase; KAP1: KRAB-associated protein 1; PML: promyelocytic leukemia; siRNA: small interfering RNA; snRNP: small nuclear ribonucleic particle; StUbL: SUMO-targeted ubiquitin-ligase; SUMO: small ubiquitin-related modifier; UIp/SENP: ubiquitin-like protease/ sentrin-specific protease; USPL1: ubiquitin-specific peptidase-like protein 1.}

\section{Acknowledgments}

We thank all members of our institute for support and stimulating discussions. This work was funded by the DFG Priority program SPP1365, SFB684, SFB815 and LOEWE Ub-Net.

Published online: 31 July 2014

\section{References}

1. Flotho A, Melchior F: Sumoylation: a regulatory protein modification in health and disease. Annu Rev Biochem 2013, 82:357-385.

2. Gareau JR, Lima CD: The SUMO pathway: emerging mechanisms that shape specificity, conjugation and recognition. Nat Rev Mol Cell Biol 2010, 11:861-871

3. Li SJ, Hochstrasser M: A new protease required for cell-cycle progression in yeast. Nature 1999, 398:246-251.

4. Li SJ, Hochstrasser M: The yeast ULP2 (SMT4) gene encodes a novel protease specific for the ubiquitin-like Smt3 protein. Mol Cell Biol 2000, 20:2367-2377

5. Berdnik D, Favaloro V, Luo L: The SUMO protease Verloren regulates dendrite and axon targeting in olfactory projection neurons. J Neurosci 2012, 32:8331-8340.

6. Bhaskar V, Smith M, Courey AJ: Conjugation of Smt3 to dorsal may potentiate the Drosophila immune response. Mol Cell Biol 2002, 22:492-504

7. Yeh ET, Gong L, Kamitani T: Ubiquitin-like proteins: new wines in new bottles. Gene 2000, 248:1-14
8. Gan-Erdene T, Nagamalleswari K, Yin L, Wu K, Pan ZQ, Wilkinson KD: Identification and characterization of DEN1, a deneddylase of the ULP family. J Biol Chem 2003, 278:28892-28900.

9. Mendoza HM, Shen LN, Botting C, Lewis A, Chen J, Ink B, Hay RT: NEDP1, a highly conserved cysteine protease that deNEDDylates Cullins. J Biol Chem 2003, 278:25637-25643.

10. Shin EJ, Shin HM, Nam E, Kim WS, Kim JH, Oh BH, Yun Y: DesUMOylating isopeptidase: a second class of SUMO protease. EMBO Rep 2012, 13:339-346.

11. Schulz S, Chachami G, Kozaczkiewicz L, Winter U, Stankovic-Valentin N, Haas P, Hofmann K, Urlaub H, Ovaa H, Wittbrodt J, Meulmeester E, Melchior F: Ubiquitin-specific protease-like 1 (USPL1) is a SUMO isopeptidase with essential, non-catalytic functions. EMBO Rep 2012, 13:930-938.

12. Mossessova E, Lima CD: Ulp1-SUMO crystal structure and genetic analysis reveal conserved interactions and a regulatory element essential for cell growth in yeast. Mol Cell 2000, 5:865-876.

13. Reverter D, Lima CD: A basis for SUMO protease specificity provided by analysis of human Senp2 and a Senp2-SUMO complex. Structure 2004, 12:1519-1531

14. Reverter D, Lima CD: Structural basis for SENP2 protease interactions with SUMO precursors and conjugated substrates. Nat Struct Mol Biol 2006, 13:1060-1068

15. Shen L, Tatham MH, Dong C, Zagorska A, Naismith JH, Hay RT: SUMO protease SENP1 induces isomerization of the scissile peptide bond. Nat Struct Mol Biol 2006, 13:1069-1077.

16. Shen LN, Dong C, Liu H, Naismith JH, Hay RT: The structure of SENP1SUMO-2 complex suggests a structural basis for discrimination between SUMO paralogues during processing. Biochem J 2006, 397:279-288.

17. Nishida T, Tanaka H, Yasuda H: A novel mammalian Smt3-specific isopeptidase 1 (SMT3IP1) localized in the nucleolus at interphase. Eur J Biochem 2000, 267:6423-6427.

18. Hang J, Dasso M: Association of the human SUMO-1 protease SENP2 with the nuclear pore. J Biol Chem 2002, 277:19961-19966.

19. Garvin AJ, Densham RM, Blair-Reid SA, Pratt KM, Stone HR, Weekes D, Lawrence KJ, Morris JR: The deSUMOylase SENP7 promotes chromatin relaxation for homologous recombination DNA repair. EMBO Rep 2013 14:975-983.

20. Guo C, Hildick KL, Luo J, Dearden L, Wilkinson KA, Henley JM: SENP3mediated deSUMOylation of dynamin-related protein 1 promotes cell death following ischaemia. EMBO J 2013, 32:1514-1528.

21. Klein UR, Haindl M, Nigg EA, Muller S: RanBP2 and SENP3 function in a mitotic SUMO2/3 conjugation-deconjugation cycle on Borealin. Mol Biol Cell 2009, 20:410-418.

22. Kuo ML, den Besten W, Thomas MC, Sherr CJ: Arf-induced turnover of the nucleolar nucleophosmin-associated SUMO-2/3 protease Senp3. Cell Cycle 2008, 7:3378-3387.

23. Wang $Y$, Yang J, Yang $K$, Cang H, Huang XZ, Li H, Yi J: The biphasic redox sensing of SENP3 accounts for the HIF-1 transcriptional activity shift by oxidative stress. Acta Pharmacol Sin 2012, 33:953-963.

24. Suh HY, Kim JH, Woo JS, Ku B, Shin EJ, Yun Y, Oh BH: Crystal structure of DeSI-1, a novel deSUMOylase belonging to a putative isopeptidase superfamily. Proteins 2012, 80:2099-2104.

25. Hutten S, Chachami G, Winter U, Melchior F, Lamond Al: A role for the Cajal-body-associated SUMO isopeptidase USPL1 in snRNA transcription mediated by RNA polymerase II. J Cell Sci 2014, 127:1065-1078.

26. Goeres J, Chan PK, Mukhopadhyay D, Zhang H, Raught B, Matunis MJ: The SUMO-specific isopeptidase SENP2 associates dynamically with nuclear pore complexes through interactions with karyopherins and the Nup107-160 nucleoporin subcomplex. Mol Biol Cell 2011, 22:4868-4882.

27. Takahashi Y, Mizoi J, Toh EA, Kikuchi Y: Yeast Ulp1, an Smt3-specific protease, associates with nucleoporins. J Biochem 2000, 128:723-725.

28. Zhang $H$, Saitoh $H$, Matunis MJ: Enzymes of the SUMO modification pathway localize to filaments of the nuclear pore complex. Mol Cell Biol 2002, 22:6498-6508.

29. Cubenas-Potts C, Goeres JD, Matunis MJ: SENP1 and SENP2 affect spatial and temporal control of sumoylation in mitosis. Mol Cell Biol 2013, 24:3483-3495

30. Itahana Y, Yeh ET, Zhang Y: Nucleocytoplasmic shuttling modulates activity and ubiquitination-dependent turnover of SUMO-specific protease 2. Mol Cell Biol 2006, 26:4675-4689.

31. Hickey CM, Wilson NR, Hochstrasser M: Function and regulation of SUMO proteases. Nat Rev Mol Cell Biol 2012, 13:755-766. 
32. Gong L, Yeh ET: Characterization of a family of nucleolar SUMO-specific proteases with preference for SUMO-2 or SUMO-3. J Biol Chem 2006, 281:15869-15877.

33. Haindl M, Harasim T, Eick D, Muller S: The nucleolar SUMO-specific protease SENP3 reverses SUMO modification of nucleophosmin and is required for rRNA processing. EMBO Rep 2008, 9:273-279.

34. Yun C, Wang Y, Mukhopadhyay D, Backlund P, Kolli N, Yergey A, Wilkinson $\mathrm{KD}$, Dasso M: Nucleolar protein B23/nucleophosmin regulates the vertebrate SUMO pathway through SENP3 and SENP5 proteases. I Cell Biol 2008, 183:589-595.

35. Zunino R, Braschi E, Xu L, McBride HM: Translocation of SenP5 from the nucleoli to the mitochondria modulates DRP1-dependent fission during mitosis. J Cell Biol 2009, 284:17783-17795.

36. Sharma P, Yamada S, Lualdi M, Dasso M, Kuehn MR: Senp1 is essential for desumoylating Sumo1-modified proteins but dispensable for Sumo2 and Sumo3 deconjugation in the mouse embryo. Cell Rep 2013, 3:1640-1650.

37. Bekes M, Prudden J, Srikumar T, Raught B, Boddy MN, Salvesen GS: The dynamics and mechanism of SUMO chain deconjugation by SUMOspecific proteases. J Biol Chem 2011, 286:10238-10247.

38. Mikolajczyk J, Drag M, Bekes M, Cao JT, Ronai Z, Salvesen GS: Small ubiquitinrelated modifier (SUMO)-specific proteases: profiling the specificities and activities of human SENPs. J Biol Chem 2007, 282:26217-26224.

39. Kolli N, Mikolajczyk J, Drag M, Mukhopadhyay D, Moffatt N, Dasso M, Salvesen G, Wilkinson KD: Distribution and paralogue specificity of mammalian deSUMOylating enzymes. Biochem J 2010, 430:335-344.

40. Drag M, Mikolajczyk J, Krishnakumar IM, Huang Z, Salvesen GS: Activity profiling of human deSUMOylating enzymes (SENPS) with synthetic substrates suggests an unexpected specificity of two newly characterized members of the family. Biochem J 2008, 409:461-469.

41. Lima CD, Reverter D: Structure of the human SENP7 catalytic domain and poly-SUMO deconjugation activities for SENP6 and SENP7. J Biol Chem 2008, 283:32045-32055.

42. Shen LN, Geoffroy MC, Jaffray EG, Hay RT: Characterization of SENP7, a SUMO-2/3-specific isopeptidase. Biochem J 2009, 421:223-230.

43. Jentsch S, Psakhye I: Control of nuclear activities by substrate-selective and protein-group SUMOylation. Annu Rev Genet 2013, 47:167-186.

44. Castle CD, Cassimere EK, Denicourt C: LAS1L interacts with the mammalian Rix1 complex to regulate ribosome biogenesis. Mol Biol Cell 2012, 23:716-728.

45. Finkbeiner $E$, Haindl M, Muller S: The SUMO system controls nucleolar partitioning of a novel mammalian ribosome biogenesis complex. EMBO J 2011, 30:1067-1078.

46. Finkbeiner $E$, Haindl M, Raman N, Muller S: SUMO routes ribosome maturation. Nucleus 2011, 2:527-532.

47. Raman N, Nayak A, Muller S: The SUMO system: a master organizer of nuclear protein assemblies. Chromosoma 2013, 122:475-485.

48. Chang TH, Xu S, Tailor P, Kanno T, Ozato K: The small ubiquitin-like modifier-deconjugating enzyme sentrin-specific peptidase 1 switches IFN regulatory factor 8 from a repressor to an activator during macrophage activation. J Immunol 2012, 189:3548-3556.

49. Van Nguyen T, Angkasekwinai P, Dou H, Lin FM, Lu LS, Cheng J, Chin YE, Dong C, Yeh ET: SUMO-specific protease 1 is critical for early lymphoid development through regulation of STAT5 activation. Mol Cell 2012, 45:210-221.

50. Zhang LJ, Vogel WK, Liu X, Topark-Ngarm A, Arbogast BL, Maier CS, Filtz TM, Leid $M$ : Coordinated regulation of transcription factor Bcl11b activity in thymocytes by the mitogen-activated protein kinase (MAPK) pathways and protein sumoylation. J Biol Chem 2012, 287:26971-26988.

51. Kang X, Qi Y, Zuo Y, Wang Q, Zou Y, Schwartz RJ, Cheng J, Yeh ET: SUMOspecific protease 2 is essential for suppression of polycomb group protein-mediated gene silencing during embryonic development. Mol Cell 2010, 38:191-201.

52. Huang $C$, Han $Y$, Wang $Y$, Sun $X$, Yan $S$, Yeh ET, Chen $Y$, Cang H, Li H, Shi G, Cheng J, Tang $X, Y i$ J: SENP3 is responsible for HIF-1 transactivation under mild oxidative stress via p300 de-SUMOylation. EMBO J 2009, 28:2748-2762

53. Nayak A, Viale-Bouroncle S, Morsczeck C, Muller S: The SUMO-specific isopeptidase SENP3 regulates MLL1/MLL2 methyltransferase complexes and controls osteogenic differentiation. Mol Cell 2014, 55:47-58.
54. Yan S, Sun X, Xiang B, Cang H, Kang X, Chen Y, Li H, Shi G, Yeh ET, Wang B, Wang $X, Y i J$ : Redox regulation of the stability of the SUMO protease SENP3 via interactions with CHIP and Hsp90. EMBO J 2010, 29:3773-3786.

55. Zunino R, Schauss A, Rippstein P, Andrade-Navarro M, McBride HM: The SUMO protease SENP5 is required to maintain mitochondrial morphology and function. J Cell Sci 2007, 120:1178-1188.

56. Hattersley N, Shen L, Jaffray EG, Hay RT: The SUMO protease SENP6 is a direct regulator of PML nuclear bodies. Mol Biol Cell 2011, 22:78-90.

57. Mukhopadhyay D, Arnaoutov A, Dasso M: The SUMO protease SENP6 is essential for inner kinetochore assembly. J Cell Biol 2010, 188:681-692.

58. Mukhopadhyay D, Dasso M: The fate of metaphase kinetochores is weighed in the balance of SUMOylation during S phase. Cell Cycle 2010, 9:3194-3201.

59. Dou H, Huang C, Singh M, Carpenter PB, Yeh ET: Regulation of DNA repair through deSUMOylation and SUMOylation of replication protein $\mathrm{A}$ complex. Mol Cell 2010, 39:333-345.

60. Liu X, Chen W, Wang Q, Li L, Wang C: Negative regulation of TLR inflammatory signaling by the SUMO-deconjugating enzyme SENP6. PLOS Pathog 2013, 9:e1003480.

61. Bawa-Khalfe T, Lu LS, Zuo Y, Huang C, Dere R, Lin FM, Yeh ET: Differential expression of SUMO-specific protease 7 variants regulates epithelialmesenchymal transition. Proc Natl Acad Sci U S A 2012, 109:17466-17471.

62. Bawa-Khalfe T, Yeh ET: SUMO losing balance: SUMO proteases disrupt SUMO homeostasis to facilitate cancer development and progression. Genes Cancer 2010, 1:748-752.

63. Wang Q, Xia N, Li T, Xu Y, Zou Y, Zuo Y, Fan Q, Bawa-Khalfe T, Yeh ET, Cheng J: SUMO-specific protease 1 promotes prostate cancer progression and metastasis. Oncogene 2013, 32:2493-2498.

64. Bawa-Khalfe T, Cheng J, Lin SH, Ittmann MM, Yeh ET: SENP1 induces prostatic intraepithelial neoplasia through multiple mechanisms. J Biol Chem 2010, 285:25859-25866.

65. Yamaguchi T, Sharma P, Athanasiou M, Kumar A, Yamada S, Kuehn MR Mutation of SENP1/SuPr-2 reveals an essential role for desumoylation in mouse development. Mol Cell Biol 2005, 25:5171-5182.

66. Sun Z, Hu S, Luo Q, Ye D, Hu D, Chen F: Overexpression of SENP3 in oral squamous cell carcinoma and its association with differentiation. Oncol Rep 2013, 29:1701-1706.

67. Wang RT, Zhi XY, Zhang Y, Zhang J: Inhibition of SENP1 induces radiosensitization in lung cancer cells. Exp Ther Med 2013, 6:1054-1058.

68. Huang BB, Gao QM, Liang W, Xiu B, Zhang WJ, Liang AB: Down-regulation of SENP1 expression increases apoptosis of Burkitt lymphoma cells. Asian Pac J Cancer Prev 2012, 13:2045-2049.

doi:10.1186/s13059-014-0422-2

Cite this article as: Nayak and Müller: SUMO-specific proteases/ isopeptidases: SENPs and beyond. Genome Biology 2014 15:422 